\title{
Investigation of the Nature and Mechanism of Resistive Switching in $\mathrm{TiO}_{2-\mathrm{x}}$
}

\author{
Ali Moballegh and Elizabeth C. Dickey \\ Department of Materials Science and Engineering, North Carolina State University, Raleigh, NC, USA
}

The spatial redistribution of the charged point defects under direct-current (DC) biasing can have significant implications for electroceramic device performance and lifetime [1,2]. The transport behavior of point defects is regulated by the boundary conditions of the electrodes, which can block ion transfer across the interface to varying degrees. When the electrodes are impermeable to mass transport, there will be an accumulation of point defects in the near-electrode region that can lead to significant modifications in the local electronic carrier concentrations at the interface. Such defect redistribution is responsible for the long-term increases in leakage current in many capacitor devices. On the other hand, this same phenomena can lead to resistive switching behavior via modification of the interface Schottky barrier at the reverse-biased cathode [1-4].

In $\mathrm{TiO}_{2}$, the redistribution of charged oxygen vacancies and titanium interstitials under applied electric field leads to spatial heterogeneity in the stoichiometry of $\mathrm{TiO}_{2}$. The present work aims to understand the phenomenon of defects redistribution at the mesoscopic length scale under the degradation process. Rutile single crystals are equilibrated at specific oxygen partial pressures and temperatures to define the initial defect chemistry state [5]. Platinum electrodes approximately $300 \mathrm{~nm}$ thick are deposited by a DC sputtering system to establish Schottky contacts. The samples are then subjected up to $200 \mathrm{~V} / \mathrm{cm}$ electric field, while the leakage current is continuously monitored. After electrical degradation, correlating electrical characterization measurements with electron microscopy analysis provides insight into the mesoscopic redistribution of point defects as a function of electric field and time.

After the field-degradation process, site-selective TEM samples are prepared by focused ion beam (FIB) from the electrode region. The Ti valence state and oxygen stoichiometry near the cathode is determined using monochromated EELS mapping performed in a FEI G2 Titan. Figure 1a shows a set of EELS spectra obtained from the interface up to $200 \mathrm{~nm}$ far from the interface. The onsets of the O K edge (532 $\mathrm{eV}$ ) are considered to calibrate the spectra. A chemical shift of $\mathrm{Ti} \mathrm{L}_{2,3}$ edges, of about $0.7 \mathrm{eV}$, toward lower energy is observed from the bulk interior to the interface, consistent with a lowering of the oxidation state. In addition, significant changes in the peak splitting of the $\mathrm{Ti}_{2,3}$ edges are indicative of lower oxidation states near the electrodes. The nonstoichiometry of $\mathrm{TiO} 2-\mathrm{x}$ is quantitatively measured for each spectrum (Fig. 1b) and reaches levels as low as $\mathrm{TiO}_{1.67}$, far outside the stability region of rutile TiO2-x.

Diffraction contrast and high-resolution phase-contrast images indicate that the severe nonstoichiometry in the near-electrode regions induces microstructural defects (Fig 2a) such as dislocations and planar defects. Figures $2 \mathrm{~b}$ and $\mathrm{c}$ show locally modulated structures at the interface of $\mathrm{TiO}_{2-\mathrm{x}} / \mathrm{Pt}$ consistent with a modification in the stacking sequence in the $<011>$ direction with a periodicity of $3 \mathrm{~d}_{011}$ revealed by electron diffraction. STEM images (Fig. 2c) indicate the long range ordering of oxygen vacancies into crystallographic shear planes to form Magnéli phases. The condensation of point defects into Magnéli phases at the electrodes depletes point defect concentration in the bulk. The implications of this defect redistribution and condensation processes have significant impacts on the overall I-V behavior of the material. 


\section{References:}

[1] J.R. Jameson et al, Applied Physics Letters 91(2007),

112101. [2] R. Waser et al, Advanced Materials 21 (2009),

$2632-2663$.

[3] A. Sawa et al, Materials Today 11(2008), 28-36.

[4] D.H. Kwon, et al, Nature Nanotechnology 5(2010),

148-153. [5] M. Nowotny et al, J. Phys. Chem. B, 110

(2006), 16270-16282.

[6] The authors acknowledge funding from the National Science Foundation, Grant

Number DMR -1132058.
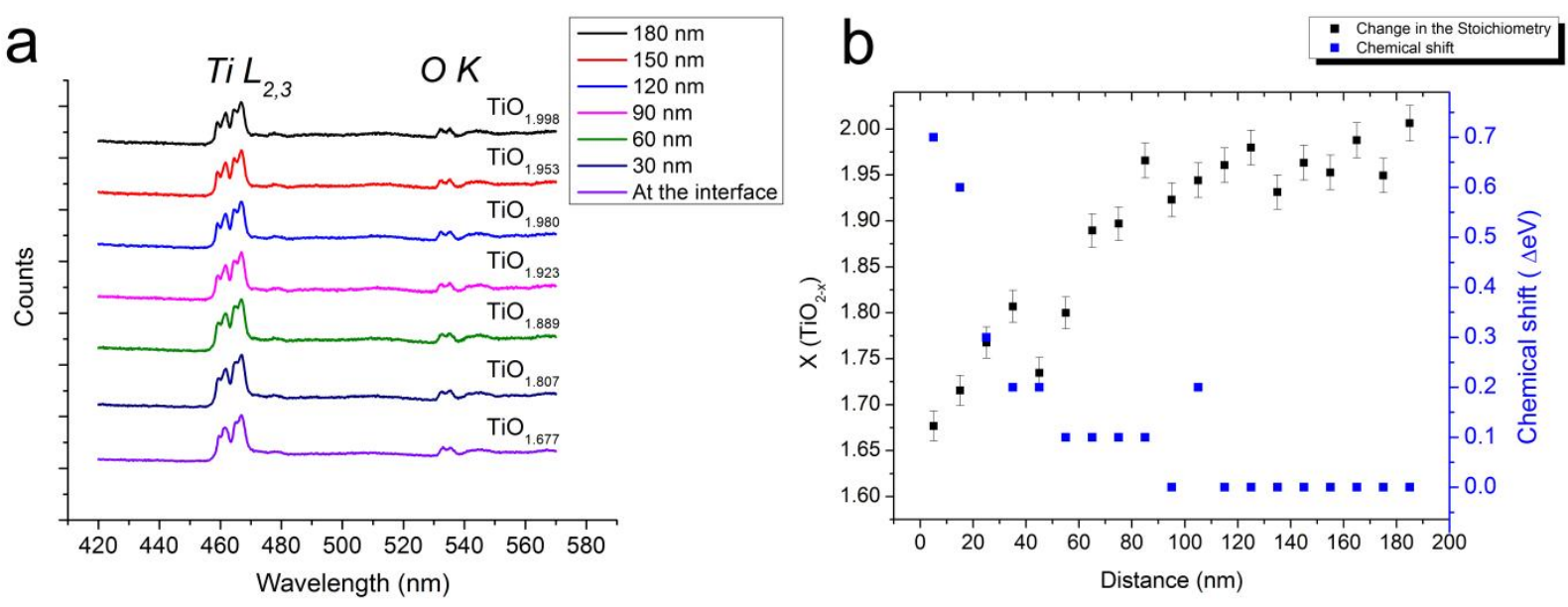

Figure 1. (a) EELS spectra of single-crystal $\mathrm{TiO}_{2}$ after electric field degradation from a region adjacent to the cathode. (b) shows the oxygen content and chemical shift of the $\mathrm{Ti}_{2,3}$ edge after degradation as a function of distance from the interface.
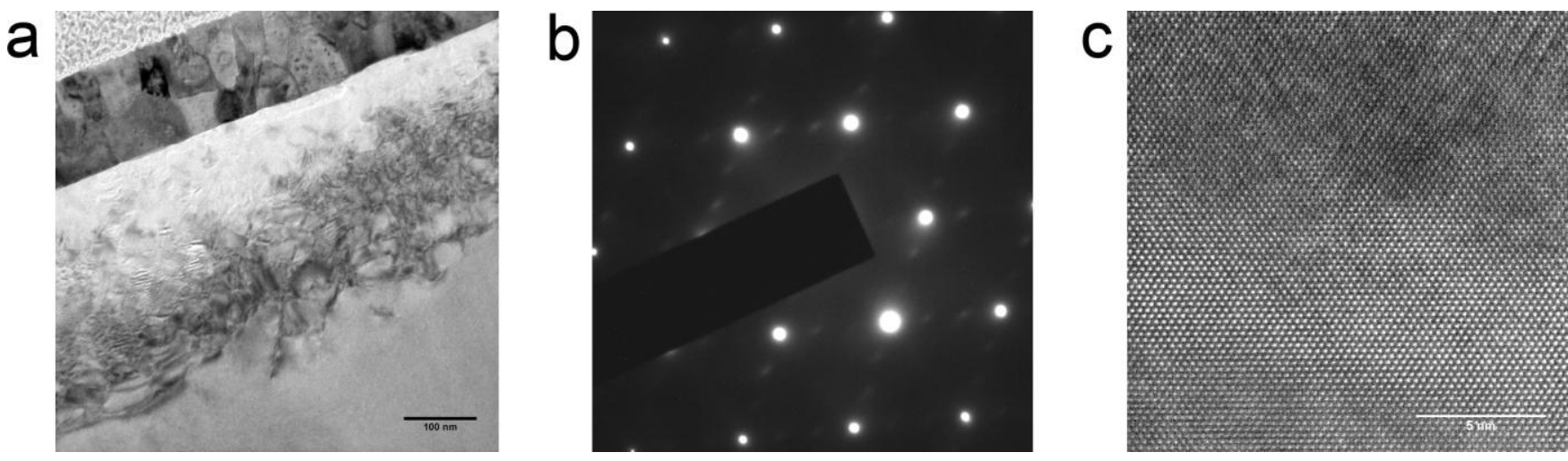

Figure 2. (a) Tem images reveal microstructural defects induced from clustering of oxygen vacancies and titanium interstitials near the cathode. (b) Diffraction patterns shows long rang ordering of underneath of the cathode after degradation with a periodicity of $3 \mathrm{~d}_{011}$. (c) STEM images indicate the condensation of point defects into crystallographic shear planes to form Magnéli phases at the electrode. 